\title{
Characterization and virulence factors distribution of blaCTX-M and mcr-1 carrying Escherichia coli isolates from bovine mastitis
}

\author{
Muhammad Shafiq ${ }^{1}$, Jinhu Huang ${ }^{2}$, Sadeeq Rahman ${ }^{3}$, Jan Shah², Chen $\mathrm{Li}^{2}$, Ilyas Ali ${ }^{2}$, and \\ Liping Wang ${ }^{2}$
}

${ }^{1}$ Affiliation not available

${ }^{2}$ Nanjing Agricultural University - Weigang Campus

${ }^{3}$ Abdul Wali Khan University Mardan

July 16, 2020

\begin{abstract}
Escherichia coli is considered as one of the critical pathogens causing contagious mastitis. The aim of the current study was to investigate the occurrence of ESBL and colistin resistant E. coli and its genotypic characterization and identification of virulence determinants in cases of bovine mastitis in Jiangsu Province, China. Five hundred and thirty-five (535) milk samples presenting mastitis symptoms were collected from three dairy farms. Susceptibility testing was identified by micro-dilution method. E. coli isolates were used to detect ESBL genes (blaCTX-M, blaSHV and blaTEM) as well as COL-resistant genes mcr-(1-9). Multiplex PCR approach was used for the detection of major CTX-M groups, different phylo-groups, and virulence genes. The clonal relationship of the isolates was then evaluated with MLST, MLSA and PFGE. Multi-drug resistance (MDR) was found in more than $85 \%$ of ESBL and/or COL resistant E. coli isolates. Genotypic characterization indicated a dominance of CTX-M-1 group, and the most prevalent alleles observed in this study were blaCTX-M-28 (38.37\%), blaCTX-M-14 (17.44\%), blaCTX-M-66 (13.95\%), blaCTX-M-55 (10.46\%), and blaCTX-M-69 (6.97\%), blaCTX-M-15 (4.65\%), blaCTX-M-177 (4.65\%), and blaCTX-M-148 (3.48\%). The results confirmed that group A was the most predominant group with $54.73 \%$, followed by group B1 and B2 $(24.21 \%, 10.52 \%)$ respectively. The targeted virulence genes were detected in $97.89 \%$ ( $\mathrm{n}=93)$ isolates. All isolates belong to group A, B1, B2, C, D, and E showed the presence of virulence factors. Among virulent genes ompC, was detected in $97.89 \%(\mathrm{n}=93)$ isolates followed by fimH in $61.05 \%(\mathrm{n}=58)$, ECs3703 57.89\% ( $\mathrm{n}=55)$, ompF 50.52\% (n=48). MLST and PFGE of the $22 \mathrm{E}$. coli isolates were determined for clonal relatedness and yielded clear fingerprint patterns. Majority of the E. coli isolates carrying ESBL and mcr-1 isolates were clonally unrelated. Sequence types 58 and ST410 were the most predominant comprising 2 each $(2 / 20=20 \%)$.
\end{abstract}

\section{ORIGINAL RESEARCH}

Shafiq et al

Title: Characterization and virulence factors distribution ofbla $a_{\mathrm{CTX}-\mathrm{M}}$ and $m c r-1$ carrying Escherichia coli isolates from bovine mastitis

Running Title: bla $a_{\mathrm{CTX}-\mathrm{M}}$ and $m c r-1 \mathrm{E}$. coli from bovine mastitis

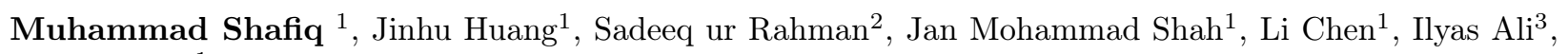
Liping Wang ${ }^{1 *}$

${ }^{1}$ MOE Joint International Research Laboratory of Animal Health and Food Safety, College of Veterinary Medicine, Nanjing Agricultural University, Nanjing 210095, China.

${ }^{2}$ College of Veterinary Sciences and Animal Husbandry, Abdul Wali Khan University, Mardan, KP, Pakistan. 
${ }^{3}$ College of Animal Sciences, Nanjing Agricultural University, Nanjing 210095, China.

\title{
* Correspondence: Liping Wang
}

MOE Joint International Research Laboratory of Animal Health and Food Safety, College of Veterinary Medicine, Nanjing Agricultural University, Nanjing 210095, China

Tel: $+86-025-2584395573$

Fax: $+86-025-84398669$

\section{E-mail: drshafiqnjau@gmail.com}

\begin{abstract}
:
Escherichia coli is considered as one of the critical pathogens causing contagious mastitis. The aim of the current study was to investigate the occurrence of ESBL and colistin resistant E. coliand its genotypic characterization and identification of virulence determinants in cases of bovine mastitis in Jiangsu Province, China. Five hundred and thirty-five (535) milk samples presenting mastitis symptoms were collected from three dairy farms. Susceptibility testing was identified by micro-dilution method. E. coli isolates were used to detect ESBL genes (bla CTX-M, bla SHV and bla TEM) as well as COL-resistant genes mcr -(1-9). Multiplex PCR approach was used for the detection of major CTX-M groups, different phylo-groups, and virulence genes. The clonal relationship of the isolates was then evaluated with MLST, MLSA and PFGE. Multi-drug resistance (MDR) was found in more than $85 \%$ of ESBL and/or COL resistant E. coliisolates. Genotypic characterization indicated a dominance of CTX-M-1 group, and the most prevalent alleles observed in this study werebla CTX-M-28 $(38.37 \%)$, bla CTX-M-14 (17.44\%), bla CTX-M-66 (13.95\%),bla CTX-M-55 (10.46\%), and bla CTX-M-69 (6.97\%), bla CTX-M-15 (4.65\%), bla CTX-M-177 (4.65\%), andbla CTX-M-148 (3.48\%). The results confirmed that group A was the most predominant group with $54.73 \%$, followed by group B1 and B2 $(24.21 \%$, $10.52 \%)$ respectively. The targeted virulence genes were detected in $97.89 \%(n=93)$ isolates. All isolates belong to group A, B1, B2, C, D, and E showed the presence of virulence factors. Among virulent genes ompC , was detected in $97.89 \%(n=93)$ isolates followed by fimH in $61.05 \%(n=58)$, ECs3703 $57.89 \%$ $(n=55)$, ompF50.52\% $(n=48)$. MLST and PFGE of the $22 \mathrm{E}$. coli isolates were determined for clonal relatedness and yielded clear fingerprint patterns. Majority of the E. coli isolates carrying ESBL and $m c r-1$ isolates were clonally unrelated. Sequence types 58 and ST410 were the most predominant comprising 2 each $(2 / 20=20 \%)$.
\end{abstract}

Keywords: Escherichia coli, Mastitis, Resistance, ESBL,

mcr-1

\section{Introduction}

Bovine mastitis is one of the most prevalent, expensive and devastating infectious diseases in the dairy industry, and poses a potential risk to public health if inadequately treated milk is consumed. Moreover, gram-negative bacteria such as E. coli is considered one of the significant pathogens of environment-linked bovine mastitis(Timofte et al., 2014). Different pathogenic possibilities exist among bovine mastitis E. coli strains. However, bovine mastitis caused byE. coli is often acute to per-acute form of the infection. The clinical manifestation of which can be from mild to fatal forms, resulting in a high incidence of mortality (Grohn et al., 2005).

In dairy animals, mastitis is one of the most important reasons for frequent and persistent use of antimicrobials. Cephalosporins and fluoroquinolones are the only antibiotics for which there is scientific evidence of valuable effects in the treatment of bovine E. colimastitis (Suojala, Kaartinen, \& Pyorala, 2013). Unfortunately, MDRE. coli strains has the potential of showing resistant to almost all antimicrobial agents in curing bovine mastitis (Eisenberger et al., 2018). Accordingly, extended-spectrum $\beta$-lactamase (ESBL)-, carbapenemase (CRE) producing E. coli is characterized by their non-susceptibility to almost all beta-lactam and 
non-beta-lactam antimicrobial agents. Meanwhile, colistin (polymyxin E), was considered as the last therapeutic option against superbugs like CRE producing E. coli. However, Liu et al. and several other studies recently reported the emergence of plasmid-mediated colistin resistant genesmcr (1-9) (Carroll et al., 2019) and characterized the colistin resistant, ESBL- and carbapenem-encoding conjugative plasmids from pigs, cattle, poultry and human beings (Liu et al., 2015). The associated resistance is due to the fact that the strain of the bacteria becomes a carrier of the factors of resistance to diverse classes of antimicrobials.

The major ESBL families includes CTX-M, SHV, TEM, OXA and other variants. The bla CтX-м $\beta$ lactamases group has been further divided into five sub groups, (bla ${ }_{\mathrm{CTX}-\mathrm{M} 1}$, bla $\mathrm{CTX}_{\mathrm{C}-\mathrm{M}-2}$, bla $\mathrm{CTX- \textrm {M } - 8}$, bla CTX-M-9, andbla CTX-M-25) and more than 120bla CTX-м enzymes have been reported (Afema et al., 2018). The success of bla СтX-M lineages has been credited to several factors, such as competent mobilization and distribution of CTX-M genes by mobile genetic elements MGEs (plasmids, transposon and insertion elements) (D'Andrea, Arena, Pallecchi, \& Rossolini, 2013). In contrast, bla CTX-M-1 andbla CTX-M-14-producing E. coli is broadly disseminated and isolated from cases of bovine mastitis in Europe (Timofte et al., 2014), whereas bla ${ }_{\text {CTX-M-15 }}$ was the most widespread ESBL type found in E. coli mastitis in Asia (Ali et al., 2017; Ohnishi et al., 2013).

Though, E. coli can be classified as pathogenic or nonpathogenic, within both of these groups there can be eight phylogenetic groups: A, B1, B2, C, D, E, and F belong to E. coli sensu stric, whereas the eight is Escherichia criptic clade I (Clermont, Christenson, Denamur, \& Gordon, 2013). Previous studies indicated that widely E. coli isolate from bovine mastitis belonged to phylo-group A (Ali et al., 2017; Suojala et al., 2011; Zhang et al., 2018). In addition, virulence genes are important for the pathogenicity of bacteria, and various virulent genes have been reported in E. coli from mastitic cows, including genes encoding aerobactin, auto agglutinating adhesion proteins, enterohemolysin, intimin, hemagglutinin, and P-fimbria (Zhang et al., 2018).

Therefore, the key objective of this study was to determine the genetic diversity of ESBL and mcr-1, antibiotic resistance rate, phylogenetic analysis and the frequency distribution of various virulent features of E. coli strains isolated from bovine clinical mastitis in Jiangsu Province, China.

\section{Material and methods}

\section{Milk samples collection}

A total of 535 milk samples with mastitis symptoms were collected from three dairy farms located in Jiangsu province during the period from May 2018 to June 2019. About $5 \mathrm{ml}$ of milk samples were aseptically collected by farm veterinarian from the affected quarter of each cow and were shipped in ice boxes to the laboratory on the same day for further microbial diagnosis.

\section{Bacterial culture, isolation, and identification ESBL phenotypes}

E. coli isolates were obtained as outlined according to the previous study (Suojala et al., 2011). Briefly the bovine mastitis milk specimens were plated on MacConkey agar, and the plates were raised $24 \mathrm{~h}$ at $37^{\circ} \mathrm{C}$. Presumptive E. coli colonies were further confirmed microscopically and verified by species specific PCR as defined earlier (Shafiq et al., 2019).

Briefly, after the preliminary growth of $E$. coli on MacConkey agar plates enriched with cefotaxime (2mg/L) and/or colistin $(2 \mathrm{mg} / \mathrm{L})$ to screen out the putative ESBL and COL-resistant isolates. The ESBL-producing $E$. coli isolates were further confirmed by a double disc synergry test (DDST) (CLSI, 2016).

\section{Antimicrobial susceptibility testing}

The antimicrobials used for susceptibility testing were ampicillin (AMP), cefotaxime (CTX), cefoxitin (CFX), ceftiofur (CEF), chloramphenicol (CHL), ciprofloxacin (CIP), colistin (COL), gentamycin (GEN), kanamycin (KEN), meropenem (MEM), polymyxin-B (POL), tetracycline (TET), and sulfamethoxazole-trimethoprim (SXT) in this study. The antimicrobial resistance (AMR) level was measured by the minimum inhibitory 
concentrations (MIC). The results were interpreted according to the principles of the Clinical and Laboratory Standards Institute (CLSI) (CLSI, 2016).

\section{DNA extraction and amplification of ESBL and COL-resistant genes}

A single colony of fresh bacterial culture from MacConkey agar was picked and inoculated into $3 \mathrm{~mL}$ of sterile Luria-Bertani (LB) medium at $37^{\circ} \mathrm{C}$ for $24 \mathrm{~h}$ in an incubator. Total DNA was extracted by a conventional boiling method. E. coli isolates were used to find out ESBL genes (bla CTX-M, bla TEM, andbla SHV) as well as COL-resistant genes mcr (1-9). All ESBL-positive isolates were further screened for the detection

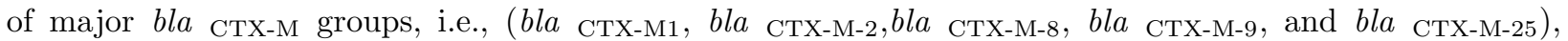
using a multiplex PCR approach with specific primers, as previously defined Table S1. PCR amplicons were sequenced in TSKINGNE Corporation (Nanjing, China). Specificbla CтX-M alleles were identified using the online database for Genomic Epidemiology (https://cge.cbs.dtu.dk/services/ResFinder/), which is using to detect acquired AMR and/or chromosomal mutations in sequenced isolates of bacteria.

\section{Phylogenetic typing}

The E. coli isolates can be classified into eight phylo-groups: seven (A, B1, B2, C, D, E, and F). Unlike isolates were assigned to the corresponding groups based on four genetic markers, namely arp $A, y j a A, \operatorname{chuA}$ , and TspE4.C2 . The phylogenetic grouping of the $E$. coli isolates was carried out by using a quadruplex PCR reaction (Clermont et al., 2013). All the primer sequences and PCR conditions are given in Table S1.

\section{Detection of virulence genes}

Virulence genes for this study were chosen based on those previously reported in $E$. coli isolates from clinical bovine mastitis. The virulence linked genes were tested by PCR were: ompC, fimH, Ecs3703, fyuA, eaeA, ler, iucD, ompF, colV, and irp2 as previously defined (Zhang et al., 2018). All the primer sequences and PCR conditions for the virulence encoding genes are listed in Table S2.

\section{Multilocus sequence typing (MLST)}

Evolutionary affiliation of the analyzed E. coli isolates was determined through MLST for which all seven householding genes ( $a d k$, fum $C$, gyrB, icd, $m d h$, purA and recA ) were amplified by PCR and sequenced following the guidelines of the MLST databases (http://mlst.warwick.ac.uk/mlst/dbs/Ecoli/) Table S3 (Wirth et al., 2006). Sequences yielded by the PCR amplification of each individual locus were utilized to obtain allelic number using online database https://pubmlst.org/bigsdb?=pubmlst_escherichia_seqdef) followed by sequence type (ST) mapping according to E. coli MLST scheme according to the reference website (https://enterobase.warwick.ac.uk/species/index/ecoli). A minimum spanning tree was built with PHYLOViZ 2.0 software (Instituto de Microbiologia, Portugal) according to the associations among MLST alleles (Ribeiro-Goncalves, Francisco, Vaz, Ramirez, \& Carrico, 2016) and to visualize the genetic relatedness and diversity of different isolates. The clonal complexes of STs were determined through goeBURST algorithm as proposed earlier (Feil, Li, Aanensen, Hanage, \& Spratt, 2004). This goeBURST algorithm is designed on a model based on bacterial evolution. Similarly, the association among the STs made in this study with the available STs in the MLST data base (https://pubmlst.org/bigsdb) was also evaluated through goeBURST (Francisco, Bugalho, Ramirez, \& Carrico, 2009). Sequence alignment of all the examined strains was performed using MUSL implemented in MEGA 7 and the maximum probability tree constructed using General Time Reversible model, Gamma distributed and invariant sites $(\mathrm{G}+1)$ with 1000 bootable replicas using MEGA version 7.0 (Tamura, Stecher, Peterson, Filipski, \& Kumar, 2013).

\section{Xbal pulsed field gel electrophoresis (Xbal-PFGE)}

To know the genetic relatedness, DNA fingerprinting profiles of the $22 m c r-1$-positive $E$. coli isolates were determined by Xbal -PFGE typing, as previously described (PulseNet, 2017).Salmonella enterica serotype Braenderup H9812 standard was used as a size marker to adjust the sizes of each fragment of XbaI -digested genomic DNA of donor isolates, especially those isolates from different gels. Fragments patterns were analyzed using BioNumerics software 7.6 (Applied-Maths, Kortrijk, Belgium), and a dendogram was generated on the 
basis of Dice coefficient with $1.0 \%$ of tolerance in the band position and $1.5 \%$ optimization. A similarity level of $80 \%$ was considered as the criterion for identifying genetically related isolates (Tenover et al., 1995).

\section{Results}

\section{Isolation and prevalence of ESBL- and COL-resistant $E$. coli}

A total of $430(80.37 \%)$ E. coli isolates were recovered from five-hundred and thirty-five clinical mastitis milk samples based on colony morphology and growth on MacConkey agar plates. Among these isolates, $325(75.58 \%)$ were confirmed as ESBL producing- $E$. coli. The maximum percentage of ESBL-producing $E$. coli isolates was detected in Farm B 147/165 (89.09\%), followed by Farm C 103/137 (75.18\%), whereas the detection rate in Farm A was 75/128 (58.59\%). Furthermore, COL-resistant isolates in Farm B was prevalent 119/165 (72.12), followed by Farm C and Farm A 97/137 (70.80\%) and 86/128 (67.18\%), respectively, as shown in Table 1.

\section{Antimicrobial susceptibility}

All the ESBL-producing E. coli isolates were tested against 13 commonly used antibiotics Figure 1. MIC testing $(\mu \mathrm{g} / \mathrm{mL})$ for all ESBL-producing E. coli were against all used antibiotics. Of the 325 E. coli isolates, the highest proportions of resistance were found against CFX $(98.15 \%)$ followed by CTX (96\%), CEF (94.76\%), AMP (93.84\%), TET (92.61\%), SXT (86.76\%), CHL (76.30\%), COL (71.07\%), POL (69.84\%), KEN (69.84\%), GEN (66.15\%), CIP (49.53\%), and MEM were found (100\%) susceptible to all these isolates. MDR was observed in more than $85 \%$ of isolates (resistance to three or more than three classes of antibiotics) by MIC.

\section{Genotyping of ESBL and COL-resistant genes}

For further molecular characterization, 95 isolates were randomly selected among these ESBL-producing $E$. coli isolates and were investigated for ESBL- and COL-resistant E. coli. Genotyping of these isolates by PCR assay showed that among ESBL determinantsbla CTX-M was the most common $(\mathrm{n}=86)$, followed bybla TEM $(\mathrm{n}=32)$ and $b l a$ SHV $(\mathrm{n}=30)$. While in COL-resistant genes only $m c r-1$ was identified and prevalent $(\mathrm{n}=75)$ in these isolates. Thebla TEM and bla SHV genes were most commonly detected in combination withbla CTX-M, and $m c r-1$ Figure $2 \mathrm{~A}$.

\section{Detection of CTX-M groups using PCR}

All CTX-M positive MDR E. coli isolates were further tested for the detection of major CTX-M groups,

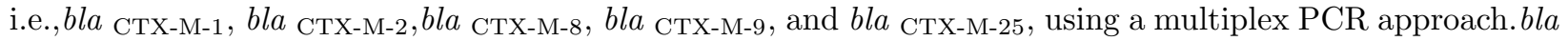
CTX-M-1 was the most common group (87\%), followed by bla CTX-M-9 $(16.27 \%)$ were found in CTX-M Positive

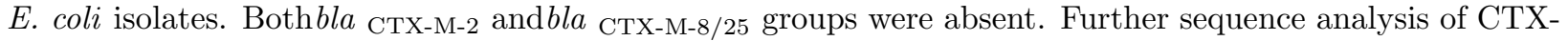
M genotypes showed thatbla CTX-M-28 was predominant allele, which was carried by $33(38.37 \%)$ isolates, followed bybla CTX-M-14 $(n=15,17.44 \%)$,bla CTX-M-66 $(n=12,13.95 \%)$, bla CTX-M-55 $(n=9,10.46 \%)$, bla CтХ-м-69 $(n=6,6.97 \%), b l a$ CTX-M-15 $(n=4,4.65 \%)$, bla CтX-M-177 $(n=4,4.65 \%)$, and bla CTX-M-148 $(n=3$, $3.48 \%$ ). Figure 2B. shows the frequency (\%) of various ESBL-encoding genes among 86 positive CTX-M $E$. coli isolates.

\section{Phylogenetic analysis of $E$. coli isolates}

Out of 95 E. coli isolates, phylogenetic group A was the most dominant group with $54.73 \%$ (n=52), followed by group B1 24.21\% ( $\mathrm{n}=23)$, group B2 10.52\% ( $\mathrm{n}=10)$, group D $5.26 \%(\mathrm{n}=5)$, group C $2.10 \%(\mathrm{n}=2)$, and one isolate was positive $1.05 \%(\mathrm{n}=1)$ which were assigned to phylogenetic group E. None of the isolates were found to assign to group F. The remaining two isolates were negative for all the genes. Details of each phylogenetic group presenting unlike gene pattern are given in Table 2.

\section{Frequency of virulence genes}

The targeted virulence genes were found in $97.89 \%(n=93)$ isolates, while two isolates were found negative for the virulent genes. Majority of the isolates belong to group A, B1, B2, C, D, and E showed the presence 
of virulence factors. Of these 95 isolates the most predominant gene was ompC , detected in $97.89 \%$ ( $n=93$ ) isolates followed by $\mathrm{fimH}$ in $61.05 \%(n=58)$, ECs3703 $57.89 \%(n=55)$, ompF $50.52 \%(n=48), i r p 231.57 \%$ $(n=30)$, fyuA $21.05 \%(n=20), i u c D 15.78 \%(n=15)$, and $2.10 \%(n=2)$ isolates were positive for colV . Two virulence genes eae $A$ and lerwere not detected in any of these isolates. The incidence of virulence genes among $E$. coli isolated from bovine clinical mastitis is shown in Table 3.

\section{Combination patterns of virulence genes}

Sixteen different gene patterns were observed in ninety-five $E$. coli isolates which are shown in Table 4 . The most prevalent pattern of four different virulence genes ompC, ompF, fimH, andECs3703 was detected in thirteen different isolates, followed by the combination of six different virulent factors ompC, ompF , ECs3703, fimH , irp2, fyuA which was detected in eight isolates.

\section{MLST and population structure}

A total of 22 isolates were organized into a total of 20 sequence types (STs) with 7 groups and 15 edges [15 0 $0]$. Sites from where the isolates were got, antibiotic resistance genes, plasmid replicon, STs and antibiogram are stated in Table 5. Moreover, STs found along with allelic profile of 22 typeable $E$. coli isolates are given in Table 6 .

The total epidemiological facts of the dominant STs were based on the MLST data of the selected isolates $(n=22)$ revealed a total of 20 STs. Sequence types 58 and ST410 were the most predominant comprising 2 each $(2 / 20=20 \%)$. All other STs were present found only once. Details of distribution and abundance of STs are labelled in Figure 3A, and 3B. The most prevalent STs (ST58) and (ST410) were originated from Farm A and Farm B, respectively.

All 20 STs originated in this study were grouped into 7 BURST group and 17 based on goeBURST analysis. Interestingly, no double locus variants (DLV) were identified among all 20STs, however, a total of 4 single locus variants (SLV) were identified among all the 7 groups. The major BG (BG 0) contained 6 isolates with 6 STs (ST354, ST2737, ST2113, ST4355, ST3901, and ST3736) with a predicted founder of ST354 (Figure 3.3. C) comprised of 3 SLVs. A second BG consisted of 5 isolates and 5 STs (ST58, ST2108, ST4024, ST865, and ST3599) with ST58 as predicted founder (Figure 3.3.D). The geoBURST analysis with all 7256 STs accessible in the database including all 20 STs mentioned in this report revealed that majority of the STs were founders such as ST10 and ST58 Figure 3C, 3D, and 3E. Unpredictably, geoBURST analysis revealed that majority of the STs found in the isolates under study (ST58, ST10, ST410, and ST178 etc.) were all founders, while other such as ST2108, and ST2113 were delineated as co-founders.

\section{Multi-locus sequence analysis (MLSA)}

To precisely categorize the investigated $E$. coli species with MDR features, we applied MLSA, which is based on the phylogenetic analysis of the nucleotide sequences of the alleles used in the MLST. Therefore, by implicating the nucleotide sequences of the typed strains $(n=22)$, the phylogenetic analysis was performed using MLST information of the strains. The maximum probability tree was created using nucleotide sequences of seven loci revealed that the strains in the study were clustered into two main sub-clusters with a larger cluster of a diverse lineage Figure 4 and majority of isolates $(n=13)$ were grouped into major cluster. A small cluster comprised of 7 isolates, were originated from random places. Two of the isolates were grouped separately into an out-group indicating out liars. Furthermore, the strains were not clustered based on the source or location. The clustering together of isolates from different regions more likely indicate that correlation between the genetic background and origin of the sample could not be established.

\section{Genetic relatedness of $E$. coli isolates}

The clonal relatedness of the 22 representative $E$. coli isolates was successfully analyzed by Xbal -PFGE. All the selected E. coli isolates were typable, and yielded clear fingerprint patterns for 22 isolates Figure 5. There were 16 different clusters based on the cutoff value of $80 \% \mathrm{~N}$. Most of the isolates carrying ESBL and $m c r-1$ isolates were clonally unrelated, except for a small proportion of E. coli strains EC (002, 003) 
and EC $(021,022)$ from the same farms, which showed $100 \%$ clonal similarity Figure. 3.5. PCR-based replicon typing (PBRT) for these twenty representativeE. coli isolates which are positive for ESBL and/or $m c r-1$ carrying different plasmid types, the most prevalent Inc. types found were IncHI2 (n=11), FIB (09), FIC (07), and X4 (05).

\section{Discussion}

Nowadays, AMR is a worldwide concern and a global effort is needed to focus on the emergence of bacterial resistance in order to find new epidemiological patterns and apply suitable approaches to eradicate the infection source and to improve treatment success rate. In the present study, four-hundred and thirty $(80.37 \%)$ isolates of $E$. coli were recovered from 535 mastitic milk samples in three dairy farms. The frequency of $E$. coli isolates in the previous and recent studies differs among herds in different countries, different regions of one country, due to smaller sample size or even caused by using different detection and identification methods (Marashifard et al., 2019). In this study, the high isolation rate of E. coli suggests that farm management should be improved in Jiangsu to lower the risks of bovine mastitis, as $E$. coli and K. pneumoniae are two environmental pathogens frequently linked with poor hygiene (Breen, Green, \& Bradley, 2009; Munoz et al., 2008). In particular, this would include the strict inspection of the udder, milking procedures, where contamination with environmental pathogens occurs in dairy herds.

AMR in Gram-negative bacteria is rising globally, mostly in E. coli . More troubling are the increasing reports of MDR pathogenic $E$. coli from food-producing animals, raising worries about animal and public health (Seiffert, Hilty, Perreten, \& Endimiani, 2013). This study determines an overall $75.58 \%$ of ESBLproducing $E$. colifrom bovine mastitis, which is higher than the previous reports of ESBL from bovine mastitis in China and other parts of the world (Ali et al., 2017; Heuvelink et al., 2019; Kar et al., 2015; Zheng et al., 2019). The overall incidence of ESBL-producing E. coli isolates was much greater in Farm B (89.09\%), followed by Farm C (75.18\%), and Farm A (58.59\%). Among E. coli isolates characterized in the present study, showed high resistance to colistin 302/430 (70.23\%). Certainly, the consistent selective pressure produced by the third generation of cephalosporins and colistin in animal production stimulated this rise of ESBL resistance. Berge et al. (Berge, Atwill, \& Sischo, 2005), though, observed high resistance in dairy cows not exposed to antibiotics. They determined that an individual antimicrobial therapy could lead to selective pressure enough to establish a resistant gene poor in the herd-level bacterial population. Among ESBL-producing E. coliisolates, the highest proportions of resistance were found against CFX $(98.15 \%)$ followed by CTX (96\%), CEF (94.76\%), AMP (93.84\%), TET (92.61\%). It is interesting that none of the $E$. coli isolates were resistant to carbapenems which is in consistent with our previous report in pigs (Shafiq et al., 2019).

Ninety-five randomly ESBL-producing E. coli were selected in this study for further genotypic characterization for the major ESBL-encoding genes (CTX, SHV, and TEM) and COL-resistant genes mcr -(1-9). We found that CTX-M type was the most prevalent $90.52 \%(n=86)$, followed by TEM $33.68 \%(n=32)$ and SHV $31.57 \%(n=30)$. While in COL-resistant genes only $m c r-1$ was identified and prevalent $78.94 \%$ $(n=75)$ in these isolates. This indicates thatbla CтX-M genes are present on most dairy cows suffering from mastitis. In our findings CTX-M-1 was the most common group (87\%), followed by CTX-M-9 (16.27\%) were found in CTX-M PositiveE. coli isolates. Among CTX-M-1 (CTX-M-28 and CTX-M-66) were the most common determinants found in 33 and $12 \mathrm{E}$. coli isolates respectively. While in CTX-M-9 group the most dominant genotype found was CTX-M-14 found in 15 isolates. Our findings of higher prevalence of CTX-M genotypes in bla CTX-M-1 group, and sporadically detection of CTX-M genes belonging to other groups are consistent with the previous studies from Chinese mainland and other parts of the world (Ali et al., 2017; Coque, Baquero, \& Canton, 2008; Geser, Stephan, \& Hächler, 2012; Yu et al., 2015).

In our study, the objective was to investigate the most common phylo-groups of E. coli strains isolated from bovine mastitis, according to the new phylo-grouping method of Clermont et al. (Clermont et al., 2013). Therefore, phylogenetic analysis was carried out in this study, and the results confirmed that group A was the most prevalent group with $54.73 \%$, followed by group B1 and B2 $(24.21 \%, 10.52 \%)$ respectively. Our findings were also in consistent with the earlier studies on bovine mastitis in which phylo-groups A and/or 
B1 were found to be the most predominant phylo-groups (Ali et al., 2017; Müştak et al., 2015; Zhang et al., 2018). Similarly, several other studies indicated that both pathogenic and nonpathogenic E. coli from bovine origin were mainly assigned to phylo-group A and B1 (Henriques, Silva, Lemsaddek, Lopes-da-Costa, \& Mateus, 2014; Silva et al., 2009; Zhang et al., 2018). We also studied the occurrence of the virulence factors, and the results confirmed that phylogenetic group A exhibited higher prevalence of various combination of virulence factors. The targeted virulent factors found in $97.89 \%(n=93)$ isolates. The most predominant virulent gene detected was ompC , found in $97.89 \%(n=93)$. Sixteen different gene patterns were observed for virulence factors, and the most prevalent pattern of four different virulence genes ompC, ompF, fimH, and ECs3703 was detected in thirteen different isolates. One recent study from China (Zhang et al., 2018), also found similar phylo-grouping and incidence circulation of virulence genes in E. coli from dairy herds with mastitis. Our results are also in accordance to these findings. This is troublesome, as these commensals may serve as reservoir for the spread of both resistant and virulent factors, and could embrace virulent traits in the future.

MLST made it possible to type and establish clonal relationship of our selected isolates and MLSA further expedited us to accomplish phylogenetic association amongst the isolates and the E. coliisolates species. MLST of 22 randomly ESBL- and $m c r-1 E$. coliisolates were organized into a total of 20 sequence types (STs). Sequence types 58 and ST410 were the most predominant comprising 2 each $(2 / 20=20 \%)$. All other STs were present found only once. The geoBURST analysis of about 7256 ST available in the PubMLST database including all 20 STs mentioned in this report revealed that majority of the STs were founders such as ST10 and ST58. Unpredictably, geoBURST analysis revealed that majority of the isolates under study were belong to founders, (ST58, ST10, ST410, and ST178 etc.), while the remaining such as ST2108, ST2113 etc . were demarcated as co-founders. Interestingly, all the above founders STs found in this study were positive for $m c r-1$ which is troublesome, and may in the future hold resistant traits in the commensal E. coli. Similarly, one recent study from China also found ST410 from mastitic cows in Inner Mongolia (Ali et al., 2017). The major carrier of different STs in these 22 E. coli isolates were detected in CTX-M-1 group. Of particular concern is that the bla CTX-M-28 andbla CTX-M-14 co-harboring mcr-1 carried major of STs in these isolates. One recent study also found thatbla CTX-M-14 allele spread in different clonal populations and are now widely disseminated in China (Zheng et al., 2019). The STs found in our study were different from the previously identified STs of $m c r-1$ - positive $E$. coli strains from food producing animals and humans in China (Kuo et al., 2016). However, ST10 identified in $m c r-1$ positive E. coli isolate was similar to the previous finding of ST10 in mcr-1 isolate from cattle in Japan and Egypt (Suzuki, Ohnishi, Kawanishi, Akiba, \& Kuroda, 2016) and sequence type 58 (ST58) identify here were similar to the previous report in $m c r-1 \mathrm{E}$. coli isolate in Brazil (Sacramento et al., 2018). In our findings, two E. coli isolates EC-021 and EC-022 were identified positive for ST410 in bovine mastitis isolates and harbored CTX-M and $m c r-1$, which is in consistent with the previous reports identified in Netherland and in China (Skov \& Monnet, 2016). These findings highlight that clonal isolates of ST410 have been identified from diverse environments, animals, and humans (Falgenhauer et al., 2016; Schaufler et al., 2016) and, as we demonstrate here, in bovine mastitis milk isolates. Hence, the simultaneous spreading of the CTX-M and $m c r-1$ genes suggests that $m c r-1$ is already present in the diverse reservoirs.

Xbal -PFGE of the 22 E. coli isolates was determined and analyzed successfully. All the representative $E$. coli isolates selected for clonal relatedness yielded clear fingerprint patterns. There were 16 different clusters sharing [?] $80 \%$ similarity in PFGE pattern. Majority of the E. coli isolates carrying ESBL and $m c r-1$ isolates were clonally unrelated. Only four isolatesE. coli strains EC $(002,003)$ and EC $(021,022)$ from the same farms, which showed $100 \%$ clonal similarity and sequence types identified were ST58 and ST410 respectively. PCR-based replicon typing (PBRT) for these twenty representative $E$. coli isolates which are positive for ESBL and/or mcr-1 carrying different plasmid types, the most prevalent Inc. types found were IncHI2 (n=11), FIB (09), FIC (07), and X4 (05). A previous study, analyzed the mcr-1 carrying plasmids available in the database at GenBank and found variety of plasmid types involved in the dissemination of $m c r-1$ in $E$. coli isolates, the major plasmid types were IncI2, IncX4, IncHI2, IncFIB and IncFII (Gao et al., 2016). 


\section{Conclusion}

In conclusion, the results of this study display high level of ESBL and COL-resistant in bovine mastitis, bla CTX-M-1 was the most common group, followed by bla CTX-M-9. The major carriers of the bla CTX-M-1 andbla CTX-M-9 werebla CTX-M-28 andbla CTX-M-14 alleles, respectively. The association of ESBL-producing E. coli with $m c r-1$ is of particular concern. Phylo-group A and phylo-group B1 of E. coliwere the predominant and virulence factors were observed in these isolates. MLST and PFGE finger printing enabled us to type and establishes clonal relatedness of ESBL-producing and $m c r-1$ - positive E. coli isolates.

\section{Acknowledgments:}

This work was supported by the National Key Research and Development Program of China (2018YFD0500300), the National Natural Science Foundation of China (31702292, 31872517), the Natural Science Foundation of Jiangsu Province (BK20170710), and the China Postdoctoral Science Foundation (2017M611841, 2018T110515), and the Priority Academic Program Development of Jiangsu Higher Education Institutions (PAPD).

\section{Ethical Statement}

Ethical Statement is not applicable.

\section{Data Availability Statement}

Data available from corresponding author upon reasonable request.

\section{Conflict of interest statement}

The authors report no conflicts of interest in this work.

\section{References}

Afema, J. A., Ahmed, S., Besser, T. E., Jones, L. P., Sischo, W. M., \& Davis, M. A. (2018). Molecular Epidemiology of Dairy Cattle-Associated Escherichia coli Carrying blaCTX-M Genes in Washington State. Appl Environ Microbiol, 84 (6). doi:10.1128/aem.02430-17

Ali, T., Rahman, S. U., Zhang, L., Shahid, M., Han, D., Gao, J., . . . Han, B. (2017). Characteristics and genetic diversity of multi-drug resistant extended-spectrum beta-lactamase (ESBL)-producing Escherichia coli isolated from bovine mastitis. Oncotarget, 8 (52), 90144-90163. doi:10.18632/oncotarget.21496

Berge, A. C., Atwill, E. R., \& Sischo, W. M. (2005). Animal and farm influences on the dynamics of antibiotic resistance in faecal Escherichia coli in young dairy calves. Prev Vet Med, 69 (1-2), 25-38. doi:10.1016/j.prevetmed.2005.01.013

Breen, J. E., Green, M. J., \& Bradley, A. J. (2009). Quarter and cow risk factors associated with the occurrence of clinical mastitis in dairy cows in the United Kingdom. J Dairy Sci, 92 (6), 2551-2561. doi:10.3168/jds.2008-1369

Carroll, L. M., Gaballa, A., Guldimann, C., Sullivan, G., Henderson, L. O., \& Wiedmann, M. (2019). Identification of Novel Mobilized Colistin Resistance Gene mcr-9 in a Multidrug-Resistant, Colistin-Susceptible Salmonella enterica Serotype Typhimurium Isolate. MBio, 10 (3). doi:10.1128/mBio.00853-19

Clermont, O., Christenson, J. K., Denamur, E., \& Gordon, D. M. (2013). The Clermont Escherichia coli phylo-typing method revisited: improvement of specificity and detection of new phylo-groups. Environ Microbiol Rep, 5 (1), 58-65. doi:10.1111/1758-2229.12019

CLSI, C. (2016). Performance standards for antimicrobial susceptibility testing. Clinical Lab Standards Institute .

Coque, T. M., Baquero, F., \& Canton, R. (2008). Increasing prevalence of ESBL-producing Enterobacteriaceae in Europe. Euro Surveill, 13 (47). 
D'Andrea, M. M., Arena, F., Pallecchi, L., \& Rossolini, G. M. (2013). CTX-M-type beta-lactamases: a successful story of antibiotic resistance.Int J Med Microbiol, 303 (6-7), 305-317. doi:10.1016/j.ijmm.2013.02.008

Eisenberger, D., Carl, A., Balsliemke, J., Kampf, P., Nickel, S., Schulze, G., \& Valenza, G. (2018). Molecular Characterization of Extended-Spectrum beta-Lactamase-Producing Escherichia coli Isolates from Milk Samples of Dairy Cows with Mastitis in Bavaria, Germany.Microb Drug Resist, 24 (4), 505-510. doi:10.1089/mdr.2017.0182

Falgenhauer, L., Imirzalioglu, C., Ghosh, H., Gwozdzinski, K., Schmiedel, J., Gentil, K., . . . Chakraborty, T. (2016). Circulation of clonal populations of fluoroquinolone-resistant CTX-M-15-producing Escherichia coli ST410 in humans and animals in Germany. Int J Antimicrob Agents, 47 (6), 457-465. doi:10.1016/j.ijantimicag.2016.03.019

Feil, E. J., Li, B. C., Aanensen, D. M., Hanage, W. P., \& Spratt, B. G. (2004). eBURST: inferring patterns of evolutionary descent among clusters of related bacterial genotypes from multilocus sequence typing data. J Bacteriol, 186 (5), 1518-1530. doi:10.1128/jb.186.5.1518-1530.2004

Francisco, A. P., Bugalho, M., Ramirez, M., \& Carrico, J. A. (2009). Global optimal eBURST analysis of multilocus typing data using a graphic matroid approach. BMC Bioinformatics, 10 , 152. doi:10.1186/14712105-10-152

Gao, R., Hu, Y., Li, Z., Sun, J., Wang, Q., Lin, J., . . . Feng, Y. (2016). Dissemination and Mechanism for the MCR-1 Colistin Resistance.PLoS Pathog, 12 (11), e1005957. doi:10.1371/journal.ppat.1005957

Geser, N., Stephan, R., \& Hächler, H. (2012). Occurrence and characteristics of extended-spectrum $\beta$ lactamase (ESBL) producing Enterobacteriaceae in food producing animals, minced meat and raw milk. $B M C$ veterinary research, 8 (1), 21.

Grohn, Y. T., Gonzalez, R. N., Wilson, D. J., Hertl, J. A., Bennett, G., Schulte, H., \& Schukken, Y. H. (2005). Effect of pathogen-specific clinical mastitis on herd life in two New York State dairy herds.Prev Vet Med, 71 (1-2), 105-125. doi:10.1016/j.prevetmed.2005.06.002

Henriques, S., Silva, E., Lemsaddek, A., Lopes-da-Costa, L., \& Mateus, L. (2014). Genotypic and phenotypic comparison of Escherichia coli from uterine infections with different outcomes: clinical metritis in the cow and pyometra in the bitch. Vet Microbiol, 170 (1-2), 109-116.

Heuvelink, A. E., Gonggrijp, M. A., Buter, R. G. J., Ter Bogt-Kappert, C. C., van Schaik, G., Velthuis, A. G. J., \& Lam, T. (2019). Prevalence of extended-spectrum and AmpC beta-lactamase-producing Escherichia coli in Dutch dairy herds. Vet Microbiol, 232 , 58-64. doi:10.1016/j.vetmic.2019.04.005

Kar, D., Bandyopadhyay, S., Bhattacharyya, D., Samanta, I., Mahanti, A., Nanda, P. K., . . . Singh, R. K. (2015). Molecular and phylogenetic characterization of multidrug resistant extended spectrum betalactamase producing Escherichia coli isolated from poultry and cattle in Odisha, India. Infect Genet Evol, 29 , 82-90. doi:10.1016/j.meegid.2014.11.003

Kuo, S. C., Huang, W. C., Wang, H. Y., Shiau, Y. R., Cheng, M. F., \& Lauderdale, T. L. (2016). Colistin resistance gene mcr-1 in Escherichia coli isolates from humans and retail meats, Taiwan. J Antimicrob Chemother, 71 (8), 2327-2329. doi:10.1093/jac/dkw122

Liu, H., Wang, Y., Wang, G., Xing, Q., Shao, L., Dong, X., . . . Ma, L. (2015). The prevalence of Escherichia coli strains with extended spectrum beta-lactamases isolated in China. Front Microbiol, 6 , 335. doi:10.3389/fmicb.2015.00335

Marashifard, M., Karimi Aliabad, Z., Malek Hosseini, S. A. A., Darban-Sarokhalil, D., Mirzaii, M., \& Khoramrooz, S. S. (2019). Determination of antibiotic resistance pattern and virulence genes in Escherichia coli isolated from bovine with subclinical mastitis in southwest of Iran. Trop Anim Health Prod, 51 (3), 575-580. doi:10.1007/s11250-018-1726-0 
Munoz, M. A., Bennett, G. J., Ahlstrom, C., Griffiths, H. M., Schukken, Y. H., \& Zadoks, R. N. (2008). Cleanliness scores as indicator of Klebsiella exposure in dairy cows. J Dairy Sci, 91 (10), 3908-3916. doi:10.3168/jds.2008-1090

Müştak, H. K., Günaydin, E., Kaya, İ. B., Salar, M. Ö., Babacan, O., Önat, K., . . Diker, K. S. (2015). Phylo-typing of clinical Escherichia coli isolates originating from bovine mastitis and canine pyometra and urinary tract infection by means of quadruplex PCR. Veterinary Quarterly, 35 (4), 194-199.

Ohnishi, M., Okatani, A. T., Harada, K., Sawada, T., Marumo, K., Murakami, M., . . Takahashi, T. (2013). Genetic characteristics of CTX-M-type extended-spectrum-beta-lactamase (ESBL)-producing enterobacteriaceae involved in mastitis cases on Japanese dairy farms, 2007 to 2011. J Clin Microbiol, 51 (9), 3117-3122. doi:10.1128/jcm.00920-13

PulseNet, C. (2017). Standard operating procedure for PulseNet PFGE of Escherichia coli O157: H7, Escherichia coli non-O157 (STEC), Salmonella serotypes, Shigella sonnei and Shigella flexneri. PNL05. Centers for Disease Control and Prevention, Atlanta, GA. In.

Ribeiro-Goncalves, B., Francisco, A. P., Vaz, C., Ramirez, M., \& Carrico, J. A. (2016). PHYLOViZ Online: web-based tool for visualization, phylogenetic inference, analysis and sharing of minimum spanning trees. Nucleic Acids Res, 44 (W1), W246-251. doi:10.1093/nar/gkw359

Sacramento, A. G., Fernandes, M. R., Sellera, F. P., Munoz, M. E., Vivas, R., Dolabella, S. S., \& Lincopan, N. (2018). Genomic analysis of MCR-1 and CTX-M-8 co-producing Escherichia coli ST58 isolated from a polluted mangrove ecosystem in Brazil. J Glob Antimicrob Resist, 15 , 288-289. doi:10.1016/j.jgar.2018.10.024

Schaufler, K., Semmler, T., Wieler, L. H., Wohrmann, M., Baddam, R., Ahmed, N., . . . Guenther, S. (2016). Clonal spread and interspecies transmission of clinically relevant ESBL-producing Escherichia coli of ST410-another successful pandemic clone? FEMS Microbiol Ecol, 92 (1). doi:10.1093/femsec/fiv155

Seiffert, S. N., Hilty, M., Perreten, V., \& Endimiani, A. (2013). Extended-spectrum cephalosporin-resistant Gram-negative organisms in livestock: an emerging problem for human health? Drug Resist Updat, 16 (1-2), 22-45. doi:10.1016/j.drup.2012.12.001

Shafiq, M., Huang, J., Ur Rahman, S., Shah, J. M., Chen, L., Gao, Y., . . Wang, L. (2019). High incidence of multidrug-resistant Escherichia coli coharboring mcr-1 and bla (CTX-M-15) recovered from pigs.Infection and drug resistance, 12 , 2135-2149. doi:10.2147/IDR.S209473

Silva, E., Leitão, S., Tenreiro, T., Pomba, C., Nunes, T., da Costa, L. L., \& Mateus, L. (2009). Genomic and phenotypic characterization of Escherichia coli isolates recovered from the uterus of puerperal dairy cows. $J$ Dairy Sci, 92 (12), 6000-6010.

Skov, R. L., \& Monnet, D. L. (2016). Plasmid-mediated colistin resistance (mcr-1 gene): three months later, the story unfolds.Euro Surveill, 21 (9), 30155. doi:10.2807/1560-7917.es.2016.21.9.30155

Suojala, L., Kaartinen, L., \& Pyorala, S. (2013). Treatment for bovine Escherichia coli mastitis - an evidencebased approach. J Vet Pharmacol Ther, 36 (6), 521-531. doi:10.1111/jvp.12057

Suojala, L., Pohjanvirta, T., Simojoki, H., Myllyniemi, A. L., Pitkala, A., Pelkonen, S., \& Pyorala, S. (2011). Phylogeny, virulence factors and antimicrobial susceptibility of Escherichia coli isolated in clinical bovine mastitis. Vet Microbiol, 147 (3-4), 383-388. doi:10.1016/j.vetmic.2010.07.011

Suzuki, S., Ohnishi, M., Kawanishi, M., Akiba, M., \& Kuroda, M. (2016). Investigation of a plasmid genome database for colistin-resistance gene mcr-1. Lancet Infect Dis, 16 (3), 284-285. doi:10.1016/s14733099(16)00008-6

Tamura, K., Stecher, G., Peterson, D., Filipski, A., \& Kumar, S. (2013). MEGA6: Molecular Evolutionary Genetics Analysis version 6.0.Mol Biol Evol, 30 (12), 2725-2729. doi:10.1093/molbev/mst197 
Tenover, F. C., Arbeit, R. D., Goering, R. V., Mickelsen, P. A., Murray, B. E., Persing, D. H., \& Swaminathan, B. (1995). Interpreting chromosomal DNA restriction patterns produced by pulsed-field gel electrophoresis: criteria for bacterial strain typing. J Clin Microbiol, 33 (9), 2233-2239.

Timofte, D., Maciuca, I. E., Evans, N. J., Williams, H., Wattret, A., Fick, J. C., \& Williams, N. J. (2014). Detection and molecular characterization of Escherichia coli CTX-M-15 and Klebsiella pneumoniae SHV-12 beta-lactamases from bovine mastitis isolates in the United Kingdom. Antimicrob Agents Chemother, 58 (2), 789-794. doi:10.1128/aac.00752-13

Wirth, T., Falush, D., Lan, R., Colles, F., Mensa, P., Wieler, L. H., . . Achtman, M. (2006). Sex and virulence in Escherichia coli: an evolutionary perspective. Mol Microbiol, 60 (5), 1136-1151. doi:10.1111/j.13652958.2006.05172.x

Yu, T., He, T., Yao, H., Zhang, J.-B., Li, X.-N., Zhang, R.-M., \& Wang, G.-Q. (2015). Prevalence of 16S rRNA methylase gene rmtB among Escherichia coli isolated from bovine mastitis in Ningxia, China.Foodborne pathogens and disease, 12 (9), 770-777.

Zhang, D., Zhang, Z., Huang, C., Gao, X., Wang, Z., Liu, Y., . . Liu, M. (2018). The phylogenetic group, antimicrobial susceptibility, and virulence genes of Escherichia coli from clinical bovine mastitis.J Dairy Sci, 101 (1), 572-580. doi:10.3168/jds.2017-13159

Zheng, B., Feng, C., Xu, H., Yu, X., Guo, L., Jiang, X., \& Song, X. (2019). Detection and characterization of ESBL-producing Escherichia coli expressing mcr-1 from dairy cows in China. J Antimicrob Chemother, 74 (2), 321-325. doi:10.1093/jac/dky446

Table 1. Isolation and prevalence of ESBL and COL-resistantE. coli from milk samples.

\begin{tabular}{llll}
\hline Farm numbers & No. of positive $E$. coli samples (\%) & No. of ESBL-positive $E$. coli isolates $(\%)$ & No. of COL-resistant $E$. \\
\hline Farm A & $128(75.29)$ & $75(58.59)$ & $86(67.18)$ \\
Farm B & $165(97.05)$ & $147(89.09)$ & $119(72.12)$ \\
Farm C & $137(80.58)$ & $103(75.18)$ & $97(70.80)$ \\
\hline
\end{tabular}

Table 2. Phylogenetic gene pattern of $E$. coli isolates from mastitis milk samples.

Total isolates $(\mathrm{n}=95)$

Total is

Group A (54.73\%) Group B1 (24.21\%) Group B2 (10.52\%) Group C (2.10\%) Group D (5.26\%) Group E (1.05\%) 522310

+; Positive, -; Negative

Table 3. The prevalence of virulence genes among $E$. coliisolated from bovine clinical mastitis $(n=95)$.

\begin{tabular}{llll}
\hline Virulence gene & Number of isolates & \% Isolates \\
\hline ompC fimH ECs3703 ompF irp2 fyuA iucD colV eaeA ler & 93585548302015200 & 97.8961 .0557 .8950 .5231 .5721 \\
\hline
\end{tabular}

Table 4. Distribution and combination patterns of virulence genes in E. coli isolates.

No. of genes Various gene combinations

1222333344445567 ompC ompC, ompF ompF, fimH ompC, irp2 ompC, ECs3703 ompC, fyuA, irp2 ompC, o 
Table 5. Molecular diversity, MLST typing, and virulence genes of twenty-two $E$. coli strains isolated from bovine mastitis.

\begin{tabular}{llllll}
\hline Strain & Location & Farm No. & B-lactamase gene (s) & Phylogroup & Virulence gene (s) \\
\hline EC-01 & Jiangsu & A & MCR-1, TEM & B1 & OMPC \\
EC-02 & Jiangsu & A & MCR-1, CTX-M, SHV & A & ECS370, FIMH, OMPC, OMPF, FYUA, IRP2 \\
EC-03 & Jiangsu & A & MCR-1, CTX-M & A & ECS370, FIMH, OMPC, OMPF, FYUA, IRP2 \\
EC-04 & Jiangsu & A & MCR-1, CTX-M & A & ECS370, FIMH, OMPC, OMPF, IRP2 \\
EC-05 & Jiangsu & A & MCR-1, CTX-M, TEM & B1 & FIMH, OMPC, OMPF, IRP2 \\
EC-06 & Jiangsu & A & CTX-M, SHV & A & ECS370, FIMH, OMPC, OMPF, IRP2 \\
EC-07 & Jiangsu & A & MCR-1, None & B2 & OMPC \\
EC-08 & Jiangsu & A & SHV, TEM & D & ECS370, FIMH, OMPC, IRP2 \\
EC-09 & Jiangsu & B & MCR-1, CTX-M & B2 & OMPC \\
EC-10 & Jiangsu & B & MCR-1, CTX-M & A & ECS370, FIMH, OMPC, OMPF, IRP2 \\
EC-11 & Jiangsu & B & MCR-1, CTX-M, TEM & A & ECS370, FIMH, OMPC, OMPF \\
EC-12 & Jiangsu & B & CTX-M, TEM & B1 & ECS370, FIMH, OMPC, OMPF, IRP2 \\
EC-13 & Jiangsu & B & MCR-1, CTX-M & E & OMPC, FIMH \\
EC-14 & Jiangsu & B & MCR-1, SHV & A & ECS370, FIMH, OMPC, OMPF \\
EC-15 & Jiangsu & B & MCR-1, TEM & B1 & ECS370, FIMH, OMPC \\
EC-16 & Jiangsu & C & MCR-1, CTX-M & A & ECS370, FIMH, ICD, OMPC, OMPF, FYUA, IRF \\
EC-17 & Jiangsu & C & MCR-1, SHV & A & ECS370, OMPC, FYUA, IRP2 \\
EC-18 & Jiangsu & C & MCR-1, CTX-M, TEM & B1 & ECS370, FIMH, OMPC, FYUA, IRP2 \\
EC-19 & Jiangsu & C & CTX-M, TEM & A & ECS370, FIMH, OMPC, OMPF, FYUA, IRP2 \\
EC-20 & Jiangsu & C & CTX-M & B2 & ECS370, FIMH, OMPC, FYUA, IRP2 \\
EC-21 & Jiangsu & C & MCR-1, CTX-M, SHV & A & ECS370, FIMH, OMPC, FYUA, IRP2 \\
EC-22 & Jiangsu & C & MCR-1, CTX-M & C & OMPC, IRP2 \\
\hline
\end{tabular}

Table 6. E. coli isolates collection used in this study, their allele profiles and sequence type identified.

\begin{tabular}{lllllllll}
\hline ID & STs & adk & fumc & gyrb & icd & mdh & purA & recA \\
\hline EC-01 & 1080 & 6 & 4 & 7 & 9 & 7 & 7 & 56 \\
EC-02 & 58 & 6 & 4 & 4 & 16 & 24 & 8 & 14 \\
EC-03 & 58 & 6 & 4 & 4 & 16 & 24 & 8 & 14 \\
EC-04 & 1722 & 15 & 88 & 78 & 29 & 59 & 294 & 62 \\
EC-05 & 10 & 10 & 11 & 4 & 8 & 8 & 8 & 2 \\
EC-06 & 178 & 10 & 4 & 4 & 8 & 8 & 8 & 2 \\
EC-07 & 865 & 6 & 4 & 4 & 16 & 8 & 8 & 14 \\
EC-08 & 2108 & 6 & 4 & 4 & 16 & 24 & 8 & 2 \\
EC-09 & 2113 & 6 & 88 & 78 & 29 & 59 & 58 & 62 \\
EC-10 & 2491 & 10 & 11 & 4 & 8 & 7 & 8 & 2 \\
EC-11 & 2735 & 10 & 88 & 78 & 29 & 59 & 58 & 62 \\
EC-12 & 3736 & 85 & 88 & 4 & 29 & 59 & 58 & 62 \\
EC-13 & 3098 & 10 & 11 & 4 & 8 & 24 & 8 & 2 \\
EC-14 & 3124 & 10 & 4 & 4 & 8 & 8 & 7 & 2 \\
EC-15 & 354 & 85 & 88 & 78 & 29 & 59 & 58 & 62 \\
EC-16 & 3599 & 10 & 11 & 4 & 16 & 24 & 8 & 14 \\
EC-17 & 3901 & 85 & 88 & 78 & 29 & 59 & 8 & 62 \\
EC-18 & 4024 & 6 & 11 & 4 & 16 & 24 & 8 & 14 \\
EC-19 & 4355 & 85 & 88 & 78 & 29 & 59 & 58 & 14
\end{tabular}




\begin{tabular}{lllllllll}
\hline ID & STs & adk & fumc & gyrb & icd & mdh & purA & recA \\
\hline EC-20 & 4583 & 6 & 11 & 4 & 16 & 8 & 8 & 2 \\
EC-21 & 410 & 6 & 4 & 12 & 1 & 20 & 18 & 7 \\
EC-22 & 410 & 6 & 4 & 12 & 1 & 20 & 18 & 7 \\
\hline
\end{tabular}

\section{Figure legends}

Figure 1. Antibiotic susceptibility profiles of extended-spectrum beta-lactamase-producing E. coli isolates from bovine mastitis

Figure 2A. Distribution of various resistance genes in combination

Figure 2B. Frequency (\%) of various ESBL-encoding genes among positive CTX-M E. coli isolates

Figure 3. Figure 3. Sequence type diversity and population structure of ESBL-producing isolates $(n=22)$

Figure 3A. MLST of 22 E. coli isolates

Figure 3B. Pie chart representing sequence type diversity

Figure 3C-E. Population structure of resistant E. coliisolates

Figure 4. Phylogenetic analysis ESBL-producing E. coliisolates $(n=22)$ from bovine mastitis. Phylogenetic tree was constructed from deduced concatenated sequences of the MLST allelic loci with maximum likelihood method using MEGA 7.0. Scale bar is indicated.

Figure 5. Dendrogram showing XbaI -PFGE patterns of the $22 \mathrm{E}$. coli isolates from this study. The dotted line on the dendrogram indicates $80 \%$ similarity as determined by the dice coefficient analysis

\section{Hosted file}

Figures . docx available at https://authorea.com/users/342405/articles/469221-characterizationand-virulence-factors-distribution-of-blactx-m-and-mcr-1-carrying-escherichia-coliisolates-from-bovine-mastitis 